\title{
Cefepime and Acute Encephalopathy: There's More to This Story
}

\author{
Michael J. Erdman * (1) \\ (c) 2020 Springer Science+Business Media, LLC, part of Springer Nature and Neurocritical Care Society
}

Acute encephalopathy (AE), defined as either delirium or depressed level of consciousness, is common in the intensive care unit (ICU) $[1,2]$. The exact cause can be hard to determine and may often be a combination of medications, acute disease processes, metabolic derangements, and unknown causes [3]. Recent literature has sought to identify what interventions and processes place patients at a high risk of AE. Numerous medications commonly used in the intensive care unit have been associated with $\mathrm{AE}$ or changes in mental status, including fluoroquinolones, voriconazole, beta-lactam antibiotics, muscle relaxers, antipsychotics, sedatives, and anticholinergics [4-8]. Beta-lactam antibiotics, including cephalosporins, carbapenems, and penicillins, have a well-documented association with $\mathrm{AE}$, and cefepime has recently been associated with $\mathrm{AE}$ [9-11].

In this issue of Neurocritical Care, Singh and colleagues conducted a retrospective, propensity-matched case-control study to determine the association between cefepime and both the incidence and duration of $\mathrm{AE}$. A randomly selected patient population comprising patients who did and did not experience AE (delirium or depressed level of consciousness without deep sedation) were included. Patients were then matched based on age, Charlson Comorbidity Index, and 24-h Acute Physiology and Chronic Health Evaluation III (APACHE III) score. The authors noted that sepsis was not part of the matching due to its inclusion in the APACHE III score, though age is also included in that score. The authors found that shock, midazolam infusion, acute kidney injury (AKI), renal disease, and cefepime use were independently

*Correspondence: Michael.Erdman@jax.ufl.edu

UF Health Jacksonville, 655 8th St. West, C-89, Jacksonville, FL 32209, USA

This comment refers to the article available at https://doi.org/10.1007/ s12028-020-01035-W. associated with AE. Risk factors for increased AE duration were similar.

A common criticism of observational retrospective studies is the fact that biases between the treatment group and the control group often exist. Detection bias may occur in situations where knowledge of the increased risk of adverse events due to exposure causes the clinician to perform increased monitoring, increasing the likelihood of detecting the adverse effect [12]. Protopathic bias may occur when an underlying condition necessitates the use of a particular intervention, causing an association between the two that may be falsely associated with the intervention rather than the preexisting disease [12]. Confounding by indication can be broken down into disease severity bias, where certain patients may be more likely to receive a particular intervention due to their severity of illness, and channeling bias, where patients with certain comorbidity are more likely to receive an intervention [12]. Propensity score matching is a well-studied statistical method of accounting for known biases in observational studies [12]. Though the treatment effect may vary from randomized clinical trials (RCTs), they generally agree with findings from RCTs conducted on critically ill patients [13]. Unlike trials with randomized enrollment, however, propensity matching lacks the ability to balance for unknown biases and can actually exacerbate unbalanced, unmeasured variables [14].

While Singh and colleagues did an excellent job matching for some previously identified risk factors for AE, information was not provided on multiple medications and disease states that could contribute to AE, limiting the strength of their findings. No information was provided regarding the frequency of neuromonitoring, leaving the risk of detection bias unknown. A lack of information regarding the source of infection limits the ability to adjust for severity of illness other than shock.

\section{Springer}


While APACHE III score was included, it was measured in the first $24 \mathrm{~h}$ of admission, compared to the average time to onset of AE of 4 days after cefepime initiation, of which timing information was not provided. Cefepime is often used in patients at risk for resistant infections and those not responding to initial treatment. It is unknown how often the patients in this study were started on cefepime as empiric therapy or were changed to cefepime after a lack of response to a different antimicrobial. Cefepime was the only antimicrobial included in this study, though multiple other antibiotics have been associated with either $\mathrm{AE}$ or delirium. Other cephalosporins and penicillins have been associated with seizures and delirium $[9,15]$. Metronidazole has been associated with neurotoxicity and may be co-administered with cefepime to provide additional anaerobic coverage. Sulfonamides, fluoroquinolones, and macrolides have been associated with psychosis as well [9]. Piperacillin/tazobactam when given in combination with vancomycin has been associated with an increased risk of AKI, which was a stronger risk factor than cefepime for AE in this study [16].

While this study provides needed information regarding risk factors for $\mathrm{AE}$, it is still unclear how much their effect compares with other similar treatment options and patient characteristics. Future studies should aim to include all known risk factors for $\mathrm{AE}$, and large databases such as the one used by Singh et al. will be integral in comparing treatment options and identifying previously unknown obstacles to curing coma.

\section{Conflict of interest}

The author has no conflict of interest.

\section{Publisher's Note}

Springer Nature remains neutral with regard to jurisdictional claims in published maps and institutional affiliations.
Received: 1 July 2020 Accepted: 28 August 2020

Published online: 15 September 2020

\section{References}

1. Slooter AJC, Otte WM, Devlin JW, et al. Updated nomenclature of delirium and acute encephalopathy: statement of ten societies. Intensive Care Med. 2020;46(5):1020-2.

2. Ely EW. Delirium as a predictor of mortality in mechanically ventilated patients in the intensive care unit. JAMA. 2004;291(14):1753.

3. Girard TD, Pandharipande PP, Ely EW. Delirium in the intensive care unit. Crit Care. 2008;12(Suppl 3):S3.

4. Mattappalil A, Mergenhagen KA. Neurotoxicity with antimicrobials in the elderly: a review. Clin Ther. 2014:36(11):1489.e4-1511.e4.

5. Jin H, Wang T, Falcione BA, et al. Trough concentration of voriconazole and its relationship with efficacy and safety: a systematic review and meta-analysis. J Antimicrob Chemother. 2016;71(7):1772-85.

6. Shprecher D, Sloan CT, Sederholm B. Neuropsychiatric side effects of cyclobenzaprine. BMJ Case Rep. 2013;2013:bcr2013008997.

7. Pasina L, Colzani L, Cortesi L, et al. Relation between delirium and anticholinergic drug burden in a cohort of hospitalized older patients: an observational study. Drugs Aging. 2019;36(1):85-91.

8. Mehta S, Cook D, Devlin JW, et al. Prevalence, risk factors, and outcomes of delirium in mechanically ventilated adults*. Crit Care Med. 2015:43(3):557-66.

9. Bhattacharyya S, Darby RR, Raibagkar P, Castro LNG, Berkowitz AL. Antibiotic-associated encephalopathy. Neurology. 2016;86(10):963-71.

10. Payne LE, Gagnon DJ, Riker RR, et al. Cefepime-induced neurotoxicity: a systematic review. Crit Care. 2017;21(1):276.

11. Fugate JE, Kalimullah EA, Hocker SE, Clark SL, Wijdicks EFM, Rabinstein AA. Cefepime neurotoxicity in the intensive care unit: a cause of severe, underappreciated encephalopathy. Crit Care. 2013;17(6):R264.

12. Ali AK. Biases related to prescribing decisions in retrospective database research in diabetes. Value Outcomes Spotlight. 2015;1(4):13-5.

13. Kitsios GD, Dahabreh IJ, Callahan S, Paulus JK, Campagna AC, Dargin JM. Can we trust observational studies using propensity scores in the critical care literature? A systematic comparison with randomized clinical trials. Crit Care Med. 2015;43(9):1870-9.

14. Brooks JM, Ohsfeldt RL. Squeezing the balloon: Propensity scores and unmeasured covariate balance. Health Serv Res. 2013;48(4):1487-507.

15. Grahl JJ, Stollings JL, Rakhit S, et al. Antimicrobial exposure and the risk of delirium in critically ill patients 11 Medical and Health Sciences 1103 Clinical Sciences. Crit Care. 2018;22(1):1-8.

16. Gomes DM, Smotherman C, Birch A, et al. Comparison of acute kidney injury during treatment with vancomycin in combination with piperacillin-tazobactam or cefepime. Pharmacotherapy. 2014;34:662-9. 